\title{
Spectrally Efficient OFDMA Lattice Structure via Toroidal Waveforms on the Time-Frequency Plane
}

\author{
Sultan Aldirmaz, Ahmet Serbes, and Lutfiye Durak-Ata (EURASIP Member) \\ Department of Electronics and Communications Engineering, Yildiz Technical University, Yildiz, Besiktas, 34349 Istanbul, Turkey \\ Correspondence should be addressed to Sultan Aldirmaz, sultanaldirmaz@gmail.com
}

Received 2 January 2010; Accepted 29 June 2010

Academic Editor: L. F. Chaparro

Copyright (๑) 2010 Sultan Aldirmaz et al. This is an open access article distributed under the Creative Commons Attribution License, which permits unrestricted use, distribution, and reproduction in any medium, provided the original work is properly cited.

\begin{abstract}
We investigate the performance of frequency division multiplexed (FDM) signals, where multiple orthogonal Hermite-Gaussian carriers are used to increase the bandwidth efficiency. Multiple Hermite-Gaussian functions are modulated by a data set as a multicarrier modulation scheme in a single time-frequency region constituting toroidal waveform in a rectangular OFDMA system. The proposed work outperforms in the sense of bandwidth efficiency compared to the transmission scheme where only single Gaussian pulses are used as the transmission base. We investigate theoretical and simulation results of the proposed methods.
\end{abstract}

\section{Introduction}

As the demand for mobility and high performance in multimedia services increase, efficient spectrum usage becomes a critical issue in wireless communications. Orthogonal frequency division multiplexing (OFDM) is a multicarrier modulation that has been employed by a number of current and future wireless standards including $802.11 \mathrm{a} / \mathrm{g}, 802.16 \mathrm{a}-$ $\mathrm{d}$, long term evolution (LTE) downlink, and next generation networks. OFDM has been a popular method because of its robustness in frequency selective fading characteristics of broadband wireless systems. OFDM technology transmits data by dividing it into parallel streams to be modulated by subchannels each having a different carrier frequency. Basically, data is carried on narrow-band subcarriers in frequency domain and the carrier spacing is carefully selected so that each subcarrier is orthogonal to the others.

There have been numerous studies in the literature to provide efficient spectrum usage in high-performance wireless applications, including video streaming $[1,2]$. Different coding methods and modulation types are developed for this task, such as hexagonal QAM structure [3, 4]. In [5], linear combinations of Hermite-Gaussian functions are used in the generation of two orthogonal pulse shapes to increase the system throughput. However, such a linear combination increases the bandwidth of a single subchannel significantly.
Pulse shape design is an important research issue because of the corruption eects of channels, such as Doppler shifts, fading, and noise. Conventional OFDM uses rectangularpulse shapes for each data. Since this pulse shape has sinc shape in the frequency domain, its energy is dispersed to other subcarriers when the channel is dispersive. For this purpose, there is a ceaseless pursuit for dierent pulse shapes. Nyquist pulses with raised cosine spectra, Hermite-Gaussian functions based pulses $[5,6]$ and an optimized combination of Slepian sequences [7-9] have been investigated in the literature. In [7], prolate spheroidal wave functions (PSWFs) which have maximum energy concentration within a given time interval are used to design a time-frequency division multiplexing (TFDM) system for multiple users. If the transmitted pulses have maximum concentration on the joint time-frequency (T-F) domain, then the transmitted signal shall be preserved against the channel affects better. Intersymbol interference (ISI) and inter-carrier interference (ICI) are the main problems of OFDM systems. When the transmission channel is time and frequency dispersive, the transmitted signal spreads in both domains. Thus symbols corrupt each other. To avoid ISI and ICI, pulse shape design and hexagonal lattice structures are introduced. In a hexagonal lattice structure, time and frequency distances are increased, so the spread signal may not be affected by the other symbols. Ambiguity function (AF) is also a useful 


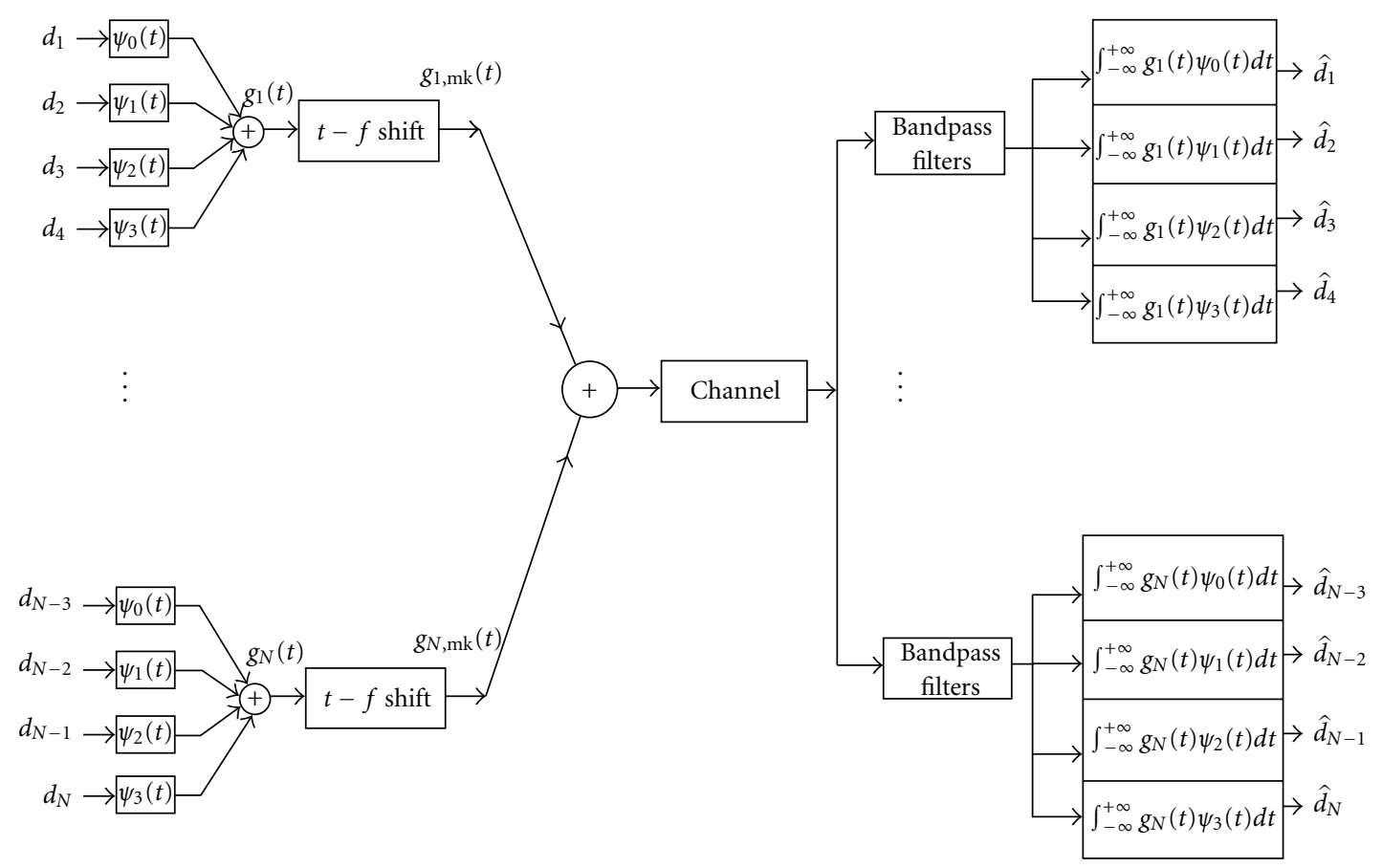

Figure 1: The proposed system model. Hermite-Gaussian functions are combined to form each symbol.

time-frequency tool to analyze ISI and ICI which provides us to observe pulse spread both in time and frequency due to the channel effects $[10,11]$. In $[5,6]$, a linear combination of Hermite-Gaussian functions is optimized to construct a desired pulse shape against the Doppler affect. In [12], Hermite functions are used in UWB communication systems with different modulation types such as PPM, BPSK, and pulse shape modulation. Linear combinations of Hermite pulses of orders 0 to 3 are obtained to construct a single pulse shape which obeys FCC constraints in [13].

In this work we propose a toroidal waveform in a rectangular lattice structure to increase the data rate by providing spectrum efficiency. We use a Hermite-Gaussian function for each data to transmit and combine $N$ of them to form a symbol. Figure 1 presents the general framework of the proposed system. For example, assuming a BPSK modulated data set $D=\left[d_{1}, d_{2}, \ldots, d_{N}\right]$, the proposed algorithm generates a pulse $g(t)=d_{1} \psi_{0}(t)+\cdots+$ $d_{i+1} \psi_{i}(t)+\cdots+d_{N} \psi_{N-1}(t)$, where $\psi_{i}(t)$ is the $i$ th order Hermite-Gaussian function. As Hermite-Gaussian functions are orthogonal to each other, the demodulation process is carried out easily.

Hermite-Gaussian functions possess doughnut-shapes on the time-frequency plane. Hence, the algorithm constitutes a toroidal waveform structure on the time-frequency plane. Figure 2 presents the waveform of the system in timefrequency plane for the baseband transmission. The figure shows time-frequency regions are occupied by HermiteGaussian functions of orders $i=0,1,2,3$. Therefore, linear combinations of them at different orders constitute a toroidal structure. Afterwards, we shift the baseband structure in both

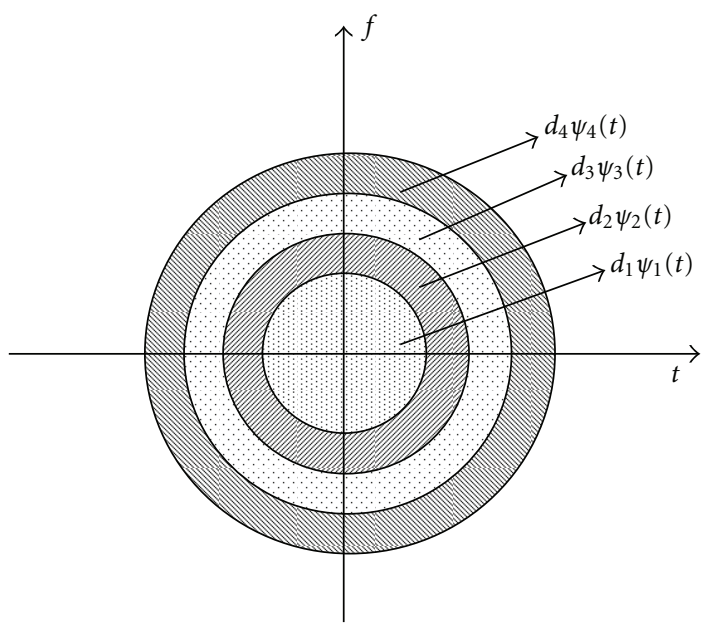

Figure 2: Toroidal-lattice structure model of a baseband transmission signal.

time and frequency to obtain the rectangular OFDM structure. Consequently, we investigate the bandwidth efficiency of the proposed system.

The remainder of this paper is organized as follows. In Section 2, a preliminary is given about Hermite-Gaussian pulses and their time-frequency localization. System model and the receiver part are described in Section 3. The simulation results are discussed by presenting both SNR versus BER values for different overlapping percentages of the signal waveforms on the lattice form in Section 4. Finally, conclusions are drawn in Section 5. 


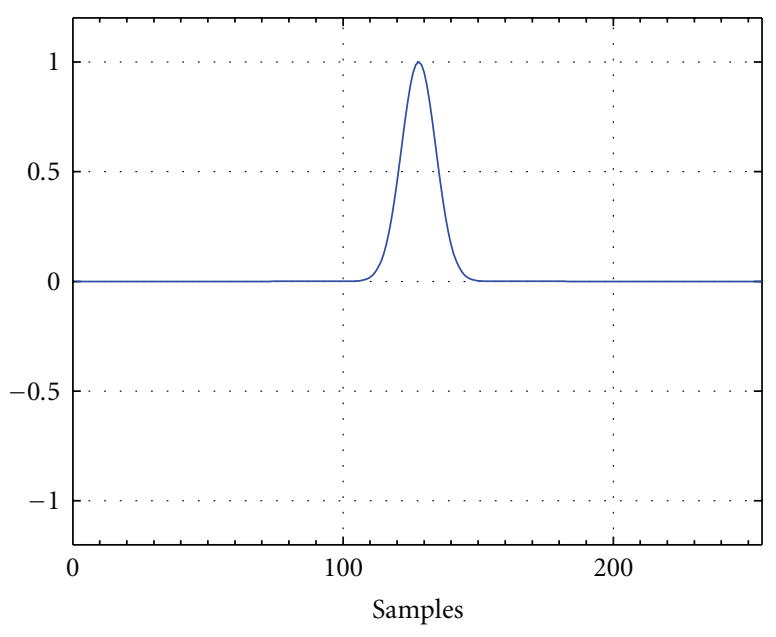

(a)

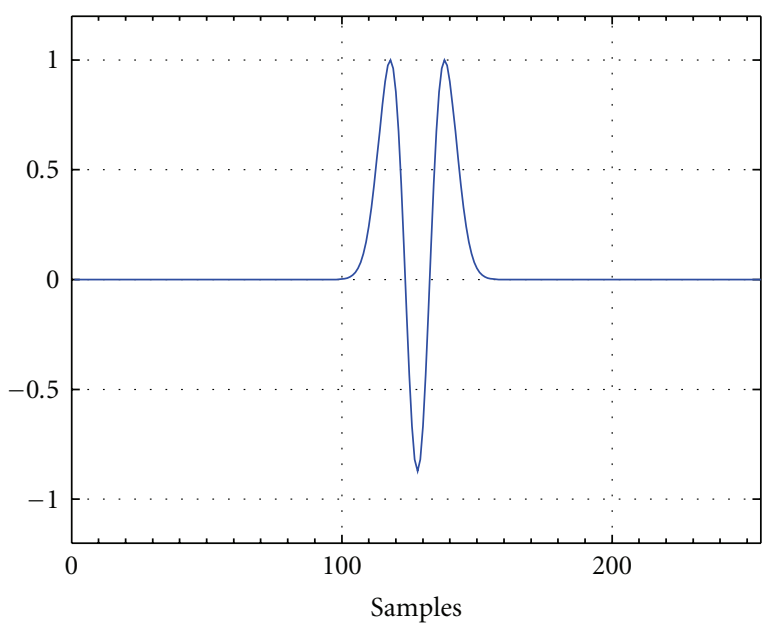

(c)

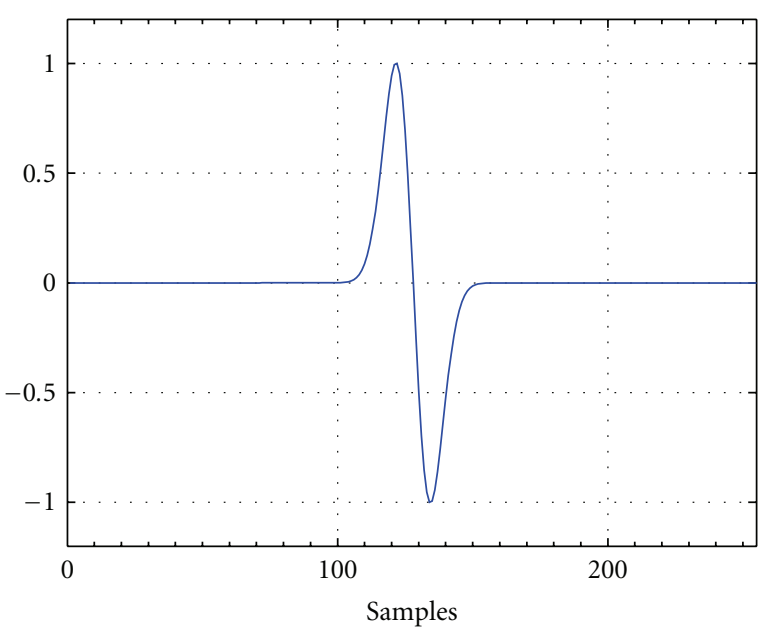

(b)

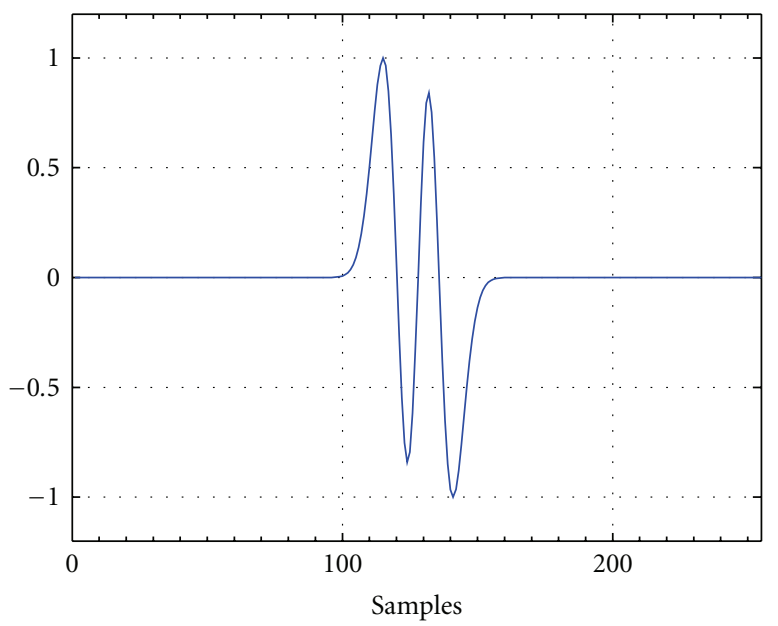

(d)

FIgURe 3: (a) 0th-order, (b) 1st-order, (c) 2nd-order and (d) 3rd-order Hermite-Gaussian pulses in time domain.

\section{Preliminaries}

2.1. Hermite-Gaussian Functions. The Hermite-Gaussian functions span the Hilbert space $L^{2}(R)$ of square-summable functions. They are well localized in both time and frequency domains. These functions are defined by a Hermite polynomial modulated with a Gaussian function as

$$
\psi_{k}(t)=\frac{2^{1 / 4}}{\sqrt{2^{k} k !}} H_{k}(\sqrt{2 \pi} t) e^{-\pi t^{2}},
$$

where $H_{k}(t)$ are the Hermite polynomial series that are expressed by

$$
H_{k}(t)=(-1)^{k} e^{t^{2}} \frac{\mathrm{d}^{k}}{\mathrm{~d} t^{k}} e^{-t^{2}}
$$

A few Hermite polynomials are as follows:

$$
\begin{gathered}
H_{0}(t)=1, \quad H_{1}(t)=2 t \\
H_{2}(t)=4 t^{2}-2, \quad H_{3}(t)=8 t^{3}-12 t, \ldots .
\end{gathered}
$$

Hermite functions are orthogonal to each other, that is, [14, page 40]

$$
\int \psi_{m}(t) \psi_{n}(t) \mathrm{d} t= \begin{cases}1, & m=n, \\ 0, & m \neq n .\end{cases}
$$

Figure 3 shows $\psi_{0}(t), \psi_{1}(t), \psi_{2}(t)$, and $\psi_{3}(t)$, respectively.

2.2. Time-Frequency Localization of Hermite-Gaussian Functions. Time-frequency support of a signal $x(t)$ can be measured by its time width and frequency domain bandwidth as

$$
\begin{gathered}
T_{x}=\frac{\left[\int\left(t-\eta_{t}\right)|x(t)|^{2} \mathrm{~d} t\right]^{1 / 2}}{\|x\|}, \\
B_{x}=\frac{\left[\int\left(f-\eta_{f}\right)|X(f)|^{2} \mathrm{~d} f\right]^{1 / 2}}{\|x\|},
\end{gathered}
$$




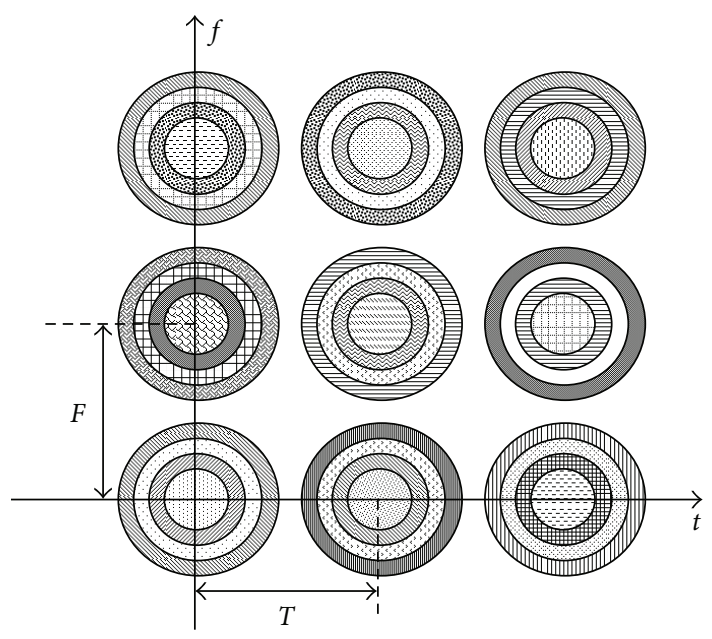

FIgURE 4: Toroidal-rectangular OFDM lattice structure. Here, F: frequency shift, T: time shift.

where $T_{x}$ and $B_{x}$ are time and frequency widths, respectively. $X(f)$ is the Fourier transform of $x(t), \eta_{t}$ and $\eta_{f}$ are time and frequency mean values defined by

$$
\begin{gathered}
\eta_{t}=\frac{\int t|x(t)|^{2} \mathrm{~d} t}{\|x\|^{2}}, \\
\eta_{f}=\frac{\int f|X(f)|^{2} \mathrm{~d} f}{\|x\|^{2}},
\end{gathered}
$$

where $\|\cdot\|$ is the norm operator. Time-bandwidth product (TBP) of a signal $x(t)$ is defined as the product of timewidth and bandwidth as $\operatorname{TBP}\{x(t)\}=T_{x} B_{x}$. Uncertainty principle dictates that there is a lower bound on the spread of the energy of a signal in both time and frequency domains together. This concentration is measured by the TBP and it is bounded by [14, page 50]

$$
T_{x} B_{x} \geq \frac{1}{4 \pi}
$$

The zeroth-order Hermite-Gaussian function, or equivalently the conventional Gaussian function, is the best localized function in both time and frequency domain having the lowest TBP equal to $1 /(4 \pi)$.

\section{The System Model}

3.1. Toroidal-Rectangular Lattice Structure. The proposed system model is considered as an OFDM system using a composite of different orders of Hermite-Gaussian functions to constitute a toroidal-waveform. Hermite-Gaussians are modulated by the data forming a toroidal lattice structure, and these pulses are shifted in time and frequency domains appropriately constructing a toroidal-rectangular lattice.
The general form of an OFDM toroidal-rectangular lattice using $N$ consecutive-order Hermite-Gaussian functions $\psi_{0}, \psi_{1}, \psi_{2}, \ldots, \psi_{N}$ is

$$
s(t)=\sum_{k=0}^{K} \sum_{m=0}^{M} \sum_{i=0}^{N} d_{m, k}(i) \psi_{i}(t-m T) \exp (j 2 \pi F k t)
$$

where $k$ and $m$ are frequency and time shifts, $i$ is the order of Hermite-Gaussian pulse, and $d_{m, k}(i)$ is the modulated data. Without losing generality, we use $N=4$ different Hermite-Gaussian functions of orders $i=0,1,2$, and 3 for practical considerations and for simplicity. For example, as the order of Hermite-Gaussian functions increase, the sampling rate should also be increased due to the highly oscillatory behavior of higher-order Hermite-Gaussian functions. Figure 4 shows an example of the proposed toroidalrectangular OFDM lattice structure. The overall structure is an $M x K$ rectangular lattice with each rectangle sheltering four toroidal regions. For this example, there are nine different rectangular time-frequency regions including an additional four different toroidal regions in each rectangle as a total of 36 regions. This means that in a unit rectangular time-frequency region, the system transmits four different modulating data, which makes up a data set for a single time-frequency region. Apart from the conventional WeylHeisenberg system, the proposed algorithm produces pulses according to the data to transmit. Namely, in case of a BPSK system if the data to be transmitted is +1 , we produce $+\psi_{i}(t)$, otherwise we produce $-\psi_{i}(t)$ and add these functions to the other Hermite-Gaussians constructed similarly. For example, let us assume a BPSK data set $D=[1,-1,-1,1]$ to be transmitted in a single rectangular region. First, $g(t)=$ $\psi_{0}(t)-\psi_{1}(t)-\psi_{2}(t)+\psi_{3}(t)$, is produced in the baseband. Then $g(t)$ is shifted in time and frequency as $g_{m, k}(t)=$ $g(t-m T) \exp \left(j k 2 \pi f_{0} t\right)$, where $m$ and $k$ represent time and frequency shifts, respectively. The algorithm transmits four data symbols simultaneously in a unit time-frequency region, so we increase the data rate carried in a single rectangular region four times, but it is obvious that the bandwidth of rectangular regions increases. In the following proposition, we show that the bandwidth of these rectangular regions increase approximately 1.63 times, which means that the bandwidth efficiency is increased up to 2.44 times compared to the single Gaussian pulse used as a transmission basis in a T-F region. As we pointed out earlier HermiteGaussian signals have the lowest TBP.

Proposition 1. TBP of linear combination of HermiteGaussian signals of order 0 to 3 is $(5-\sqrt{3}) / 2 \approx 1.634$ times greater than the zeroth-order Hermite-Gaussian signal.

Proof. Hermite-Gaussian functions are defined as in (1)-(3). Zeroth-order Hermite-Gaussian function is defined as

$$
\psi_{0}(t)=2^{1 / 4} e^{-\pi t^{2}}
$$

As the time-frequency support of a signal $x(t)$ is measured by its time domain and frequency domain bandwidths, first, we 


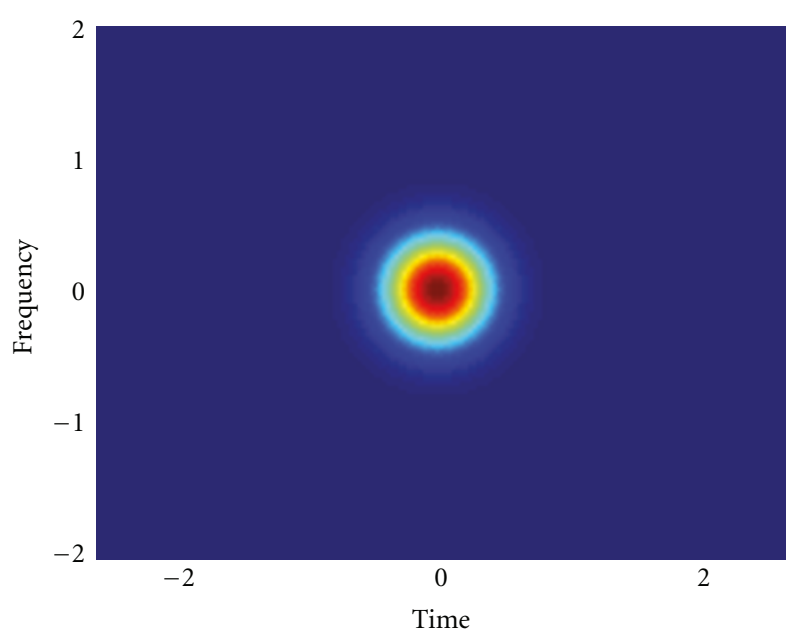

(a)

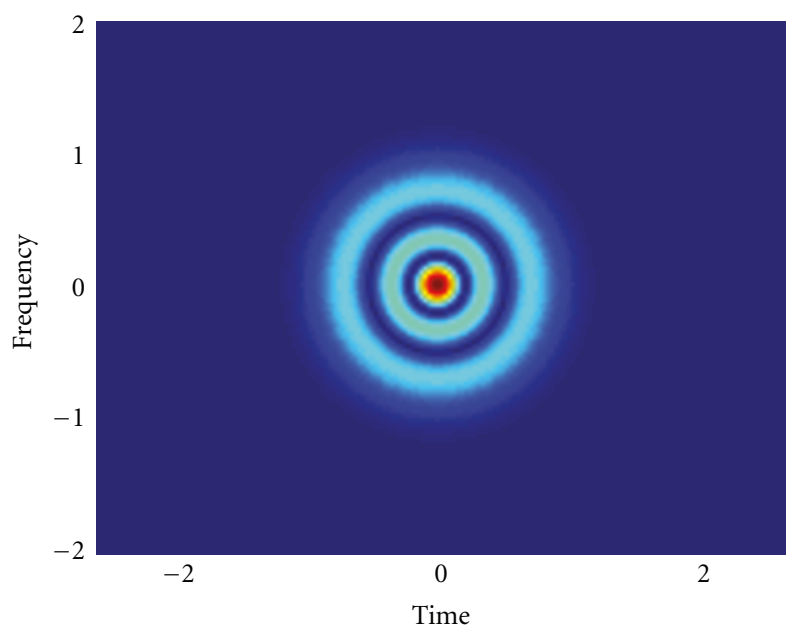

(c)

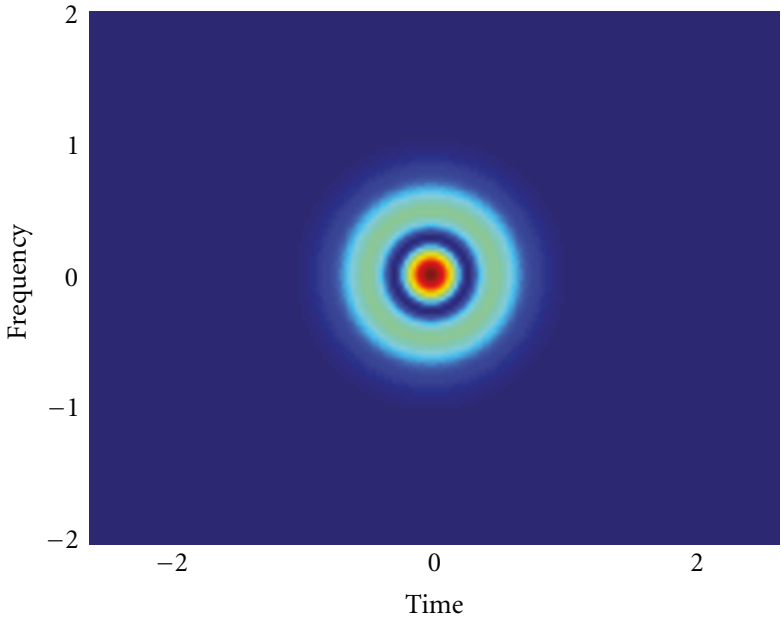

(b)

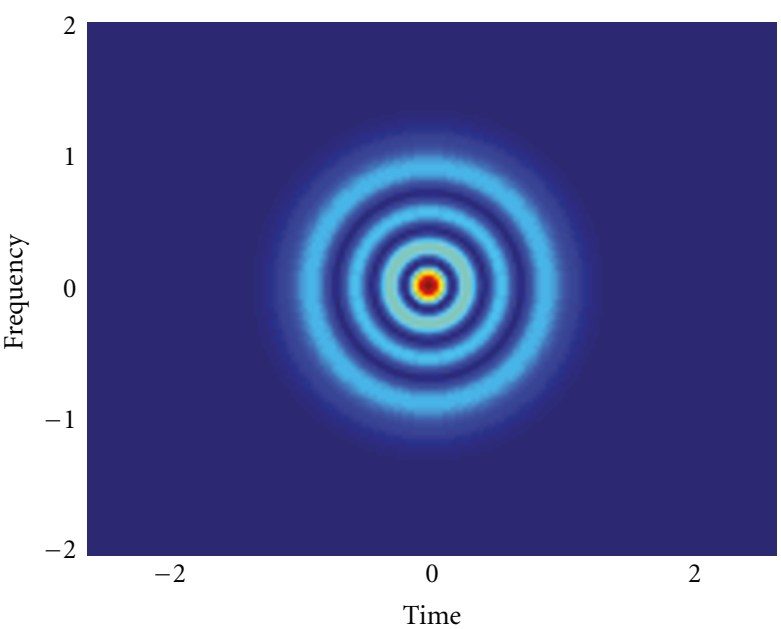

(d)

Figure 5: WD of (a) 0th-order, (b) 1st-order, (c) 2nd-order and (d) 3rd-order Hermite pulses.

calculate the mean time value of the zeroth-order HermiteGaussian function by using (7)

$$
\eta_{t}\left\{\psi_{0}\right\}=\frac{\int t\left|2^{1 / 4} e^{-\pi t^{2}}\right|^{2} \mathrm{~d} t}{\|1\|}
$$

and substitute it in (5) to find the time width as

$$
T_{x}\left\{\psi_{0}\right\}=\left[\int\left(t-\eta_{t}\left(\psi_{0}\right)\right)^{2}\left|2^{1 / 4} e^{-\pi t^{2}}\right|^{2} \mathrm{~d} t\right]^{1 / 2}=\frac{1}{2 \sqrt{\pi}}
$$

As Hermite-Gaussian functions are rotationally invariant in the time-frequency plane, it is sufficient to evaluate the timewidth only for all Hermite-Gaussian signals in order to find the TBP. The bandwidth $B_{x}$ is equal to time width $T_{x}$, for all $\psi_{k}(t)$. Therefore, TBP of the zeroth-order Hermite-Gaussian signal is defined as

$$
\operatorname{TBP}\left\{\psi_{0}\right\}=T_{x}\left\{\psi_{0}\right\} B_{x}\left\{\psi_{0}\right\}=T_{x}\left\{\psi_{0}\right\} T_{x}\left\{\psi_{0}\right\}=\frac{1}{4 \pi}
$$

In the proposed algorithm, we increase the throughput of the system by using $N$ Hermite-Gaussian functions. These pulses are added together to construct a toroidal waveform. Hermite pulses for $k=0,1,2$, and 3 are expressed as

$$
\begin{aligned}
\psi_{0}(t) & =2^{1 / 4} e^{-\pi t^{2}} \\
\psi_{1}(t) & =\frac{2^{1 / 4}}{\sqrt{2}} H_{1}(\sqrt{2 \pi} t) e^{-\pi t^{2}}=\frac{2^{1 / 4}}{\sqrt{2}} 2 \sqrt{2 \pi} t e^{-\pi t^{2}} \\
& =2^{1 / 4}(2 \sqrt{\pi}) t e^{-\pi t^{2}}
\end{aligned}
$$




$$
\begin{aligned}
\psi_{2}(t) & =\frac{2^{1 / 4}}{\sqrt{8}} H_{2}(\sqrt{2 \pi} t) e^{-\pi t^{2}}=\frac{2^{1 / 4}}{2 \sqrt{2}}\left(8 \pi t^{2}-2\right) e^{-\pi t^{2}} \\
& =2^{1 / 4}\left(\frac{4 \pi t^{2}-1}{\sqrt{2}}\right) e^{-\pi t^{2}} \\
\psi_{3}(t) & =\frac{2^{1 / 4}}{\sqrt{48}} H_{3}(\sqrt{2 \pi} t) e^{-\pi t^{2}} \\
& =\frac{2^{1 / 4}}{4 \sqrt{3}}\left(16 \pi \sqrt{2 \pi} t^{3}-12 \sqrt{2 \pi} t\right) e^{-\pi t^{2}} \\
& =2^{1 / 4}\left(\frac{4 \pi \sqrt{2 \pi} t^{3}-3 \sqrt{2 \pi} t}{\sqrt{3}}\right) e^{-\pi t^{2}}
\end{aligned}
$$

Then, the toroidal lattice structure is the sum of

$$
\begin{aligned}
\psi_{s}= & \psi_{0}+\psi_{1}+\psi_{2}+\psi_{3} \\
= & 2^{1 / 4} e^{-\pi t^{2}}+2^{1 / 4} 2 \sqrt{\pi} t e^{-\pi t^{2}}+\frac{4 \pi t^{2}-1}{2^{1 / 4}} e^{-\pi t^{2}} \\
& +2^{3 / 4} \sqrt{\pi} \frac{4 \pi t^{3}-3 t}{\sqrt{3}} e^{-\pi t^{2}} .
\end{aligned}
$$

The norm of the sum is equal to 2, when we add four orthonormal signals. Its mean time value is calculated by

$$
\eta_{t}\left\{\psi_{s}\right\}=\frac{\int_{-\infty}^{\infty} t\left|2^{1 / 4} e^{-\pi t^{2}}+2^{1 / 4} 2 \sqrt{\pi} t e^{-\pi t^{2}}+\left(\left(4 \pi t^{2}-1\right) / 2^{1 / 4}\right) e^{-\pi t^{2}}+2^{3 / 4} \sqrt{\pi}\left(\left(4 \pi t^{3}-3 t\right) / \sqrt{3}\right) e^{-\pi t^{2}}\right|^{2} \mathrm{~d} t}{2^{2}}=\frac{1+\sqrt{2}+\sqrt{3}}{4 \sqrt{\pi}}
$$

and its time width is

$$
\begin{aligned}
T_{x}\left\{\psi_{s}\right\} & =\frac{\left[\int_{-\infty}^{\infty}(t-(1+\sqrt{2}+\sqrt{6}) / 4 \sqrt{\pi})^{2}\left|2^{1 / 4} e^{-\pi t^{2}}+2^{1 / 4} 2 \sqrt{\pi} t e^{-\pi t^{2}}+\left(\left(4 \pi t^{2}-1\right) / 2^{1 / 4}\right) e^{-\pi t^{2}}+2^{3 / 4} \sqrt{\pi}\left(\left(4 \pi t^{3}-3 t\right) / \sqrt{3}\right) e^{-\pi t^{2}}\right|^{2}\right]^{1 / 2}}{2} \\
& =\frac{1}{2} \sqrt{\frac{5-\sqrt{3}}{2 \pi}}
\end{aligned}
$$

As the time width is equal to the bandwidth of the signal, TBP of the toroidal structure is

$$
\operatorname{TBP}\left\{\psi_{s}\right\}=T_{x} B_{x} \psi_{s}=\frac{5-\sqrt{3}}{8 \pi}
$$

TBP of $\psi_{s}$ compared to TBP of $\psi_{0}$ is calculated as

$$
\frac{\operatorname{TBP}\left\{\psi_{s}\right\}}{\operatorname{TBP}\left\{\psi_{0}\right\}}=\frac{T_{x} B_{x} \psi_{s}}{T_{x} B_{x} \psi_{0}}=\frac{5-\sqrt{3}}{2} \approx 1.634
$$

Consequently, we observe that the toroidal signal covers a time-frequency region of 1.63 times the region of $\psi_{0}$ pulse. However, since we transmit four pulses at a time we increase the bandwidth efficiency by $4 / 1.634 \approx 2.44$ times. Figures $5(\mathrm{a})-5(\mathrm{~d})$ show the WD of zeroth, first, second, and thirdorder Hermite-Gaussian functions, respectively. Note that Figure 5 shows time-frequency representations of HermiteGaussian functions, whose time domain representations are plotted in Figure 3. Also, it is clear that the rectangulartoroidal structure in Figure 4 is formed by the weighted sums of the Hermite-Gaussian functions with respect to the transmitted data. AF is calculated by taking the 2-D FFT of the WD. As Hermite-Gaussian functions are symmetric both in time and frequency, AF of these signals are equivalent to their WD's.
3.2. Channel Model and the Receiver Structure. The transmitted signal is sent through a channel where the received signal is modeled as

$$
r(t)=\sum_{l=1}^{L} s(t) h(l-t)+n(t)
$$

where $n(t)$ is the additive white Gaussian noise (AWGN) and $h(\cdot)$ is the channel response. The channel is assumed to have the impulse response

$$
h(t)=\sum_{p=1}^{L} \alpha_{p} e^{j \varphi_{p} t} \delta\left(t-D_{p}\right),
$$

where $L, \varphi$, and $D$ represent path number, Doppler spread and delay, respectively. In simulations, both AWGN and Rayleigh channel models are considered as the channel effect.

The proposed algorithm divides the time and frequency domains into rectangles including toroidal regions. Therefore the receiver part includes $M$ multiplicative windows to select the desired time interval and $N$ convolutional band-pass filters to separate frequency bands. Hence, we filter only a single rectangular time-frequency region in advance. Afterwards, we shift the signal to the baseband and obtain the toroidal lattice structure which contains multiple data. We take inner products of the toroidal-data with 


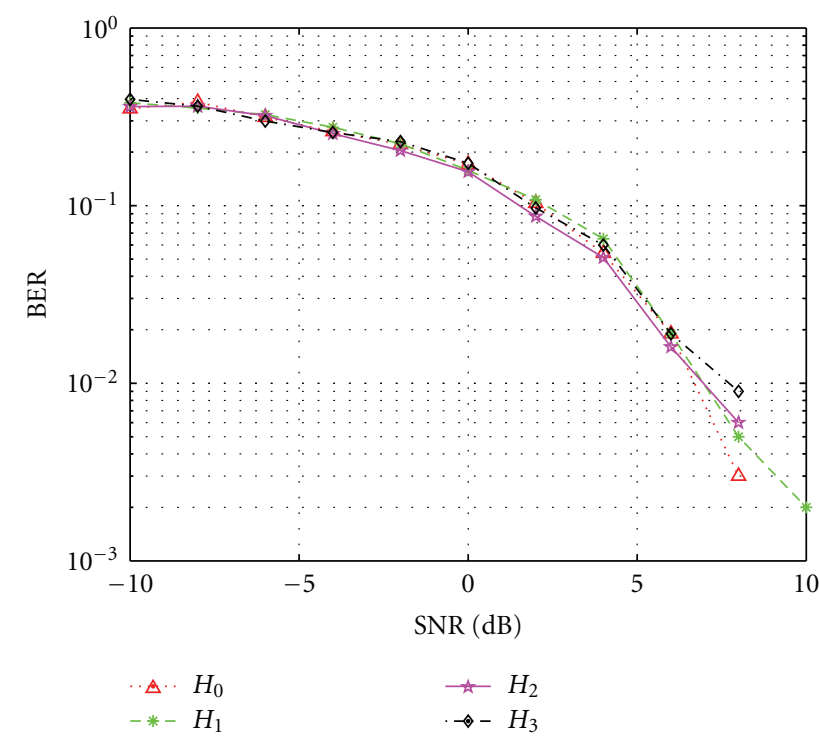

FIGURE 6: BER versus SNR for Hermite-Gaussian signals with zero to three order.

Hermite-Gaussian pulses to estimate the transmitted data by taking advantage of orthogonality between different orders of Hermite-Gaussian functions. Let us assume $r(t)=d_{1} \psi_{0}(t)+$ $d_{2} \psi_{1}(t)+d_{3} \psi_{2}(t)+d_{4} \psi_{3}(t)+n(t)$ be the received toroidal signal with AWGN $n(t)$ after filtering in time-frequency. The estimated data can be obtained as,

$$
\widehat{d}_{n}=\left\langle\psi_{n}(t), r(t)\right\rangle=\int_{-\infty}^{\infty} r(t) \psi_{n}(t) \mathrm{d} t
$$

for $n=0,1,2,3$.

Hermite-Gaussian functions and their linear combinations are orthogonal to each other. Thus, their time and frequency shifted versions are used in the proposed OFDM system simplifying the detection process.

\section{Simulation Results}

In this study, the aim is to increase the system throughput by constructing a toroidal waveform in a rectangular lattice structure. For this purpose, different orders of HermiteGaussian pulses are BPSK modulated and they are added together to generate the transmitted signal. The toroidal pulse is shifted in both time and frequency to construct a rectangular Weyl-Heisenberg system. We choose the SNR range within $[-10,10] \mathrm{dB}$ and the average of 500 MonteCarlo iterations performed in all analyses. BPSK modulation is used for the sake of simplicity. If we choose another modulation type such as M-QAM or QPSK, we may increase the throughput more. However, we do not overly concern ourselves with the modulation algorithm, since our purpose is to show the performance of the toroidal system. To examine the performances of different orders of HermiteGaussian pulses, we have used each individual HermiteGaussian as a single transmission pulse. Figure 6 presents

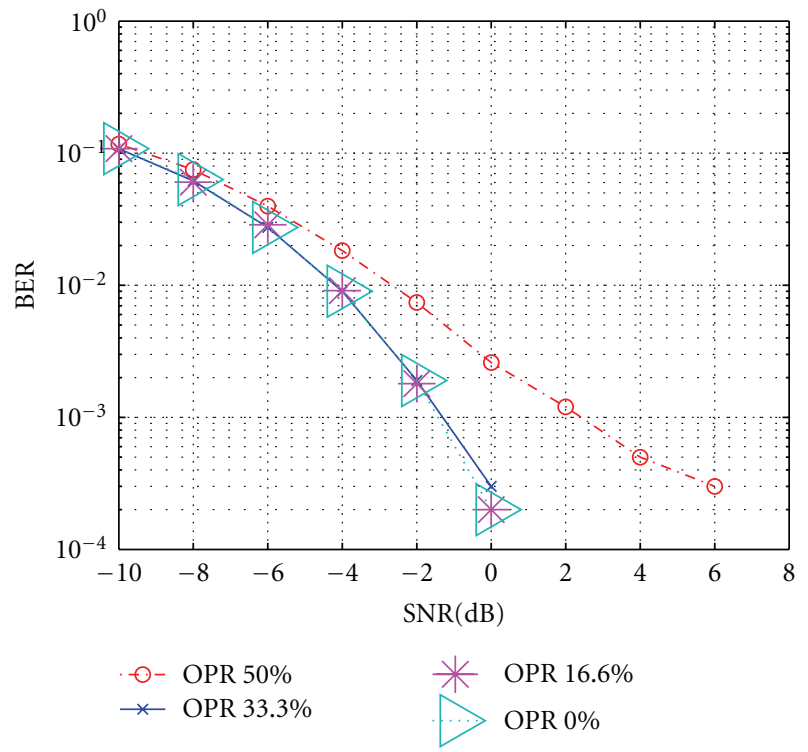

FIgURE 7: BER versus SNR with different time shifts.

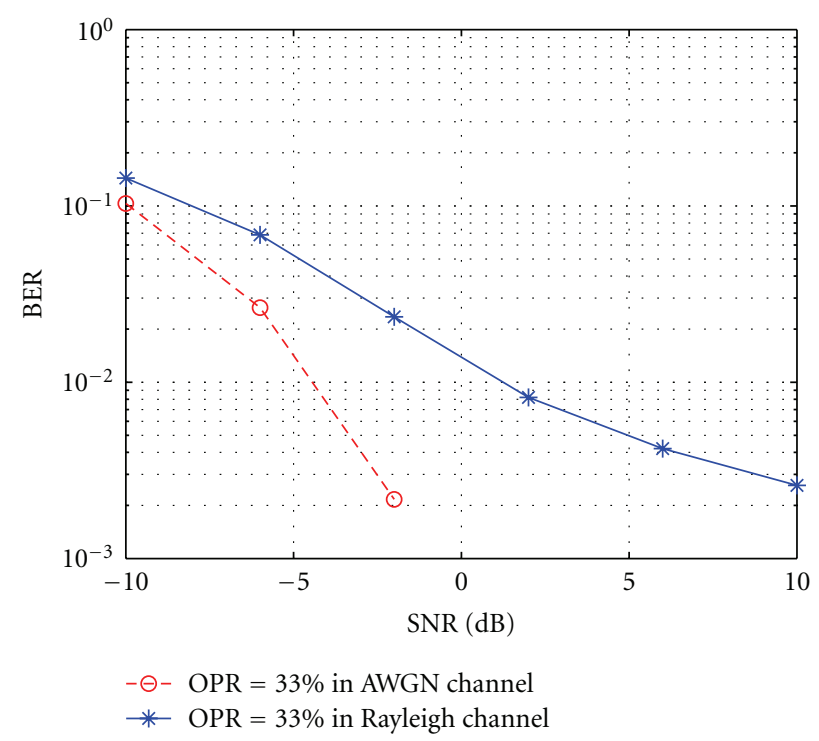

Figure 8: BER versus SNR for Rayleigh and AWGN channel.

the BER versus SNR curves for different orders of HermiteGaussian signals. Each Hermite-Gaussian signal's SNR versus BER performance is approximately equal to other ones.

The performance of the proposed system is investigated for different time distances between toroidal waveforms. We define an overlapping-pulse ratio (OPR) between adjacent pulses as the percentage of the overlapping pulses. It can be seen from Figure 7 that as the time distance is increased, or equivalently OPR is decreased, the system performance is increased. If two symbols overlap, data rate increases and the bandwidth is used more efficiently, but the system becomes more vulnerable. We have generated overlapping pulses to test how robust the proposed system is. The maximum overlap value to perfect recovery of data at the 
receiver is found to be $50 \%$ in a noiseless environment. Figure 7 presents the BER performance versus SNR of the system for different OPRs. As OPR decreases, the system performance increases. However, when OPR is small the system performance does not alter very much. We also simulate the system performance in a Rayleigh channel when the Doppler frequency is $100 \mathrm{~Hz}$ and OPR is 33.3\%.

In Figure 8, BER performance versus SNR in both Rayleigh-fading and only-AWGN channels are shown. OnlyAWGN channel is approximately $10 \mathrm{~dB}$ better than the Rayleigh-fading channel at SNR $=-2 \mathrm{~dB}$ in the BER-sense.

\section{Conclusions}

We have proposed a new toroidal waveform in a rectangularlattice OFDMA structure. The system includes $N$ differentorder Hermite-Gaussian pulses. Each of these pulses is modulated by different data, and they are combined together to construct a toroidal structure which increases the data rate up to $N$ times. However, in case of a four-layer HermiteGaussian toroidal structure, as the third-order function covers almost twice the TF region compared to zeroth-order one, the overall data rate increased more than twice. As part of future works, system robustness against doubly dispersive channel effects and ICI will be investigated.

\section{Acknowledgment}

The authors are supported by the Scientific and Technological Research Council of Turkey, TUBITAK under the grant of Project no. 105E078.

\section{References}

[1] I. Trigui, M. Siala, S. Affes, A. Stéphenne, and H. Boujemâa, "Optimum pulse shaping for OFDM/BFDM systems operating in time varying multi-path channels," in Proceedings of the 50th Annual IEEE Global Telecommunications Conference (GLOBECOM '07), pp. 3817-3821, Washington, DC, USA, November 2007.

[2] I. Kanaras, A. Chorti, M. R. D. Rodrigues, and I. Darwazeh, "Spectrally efficient FDM signals: bandwidth gain at the expense of receivercomplexity," in Proceedings of the IEEE International Conference on Communications (ICC '09), pp. 16, Dresden, Germany, June 2009.

[3] C. D. Murphy, "High-order optimum hexagonal constellations," in Proceedings of the 11th IEEE International Symposium on Personal, Indoor and Mobile Radio Communications (PIMRC '00), vol. 1, pp. 143-146, September 2000.

[4] G. Xingxin, L. Mingquan, and F. Zhenming, "Asymmetric hexagonal QAM based OFDM system," in Proceedings of the IEEE International Conference on Communications, Circuits and Systems and West Sino Expositions, vol. 1, pp. 299-302, June 2002.

[5] T. Kurt, G. K. Kurt, and A. Yongacoglu, "Throughput enhancement in multi-carrier systems employing overlapping Weyl-Heisenberg frames," in Proceedings of the IEEE International Conference on Communications (ICC '09), Dresden, Germany, June 2009.
[6] R. Ayadi, I. Kammoun, and M. Siala, "Optimization of the pulse shape of OFDM systems using the arrow-hurwicz algorithm," in Proceedings of the 4th IEEE International Symposium on Wireless Communication Systems (ISWCS '07), pp. 91-95, October 2007.

[7] S. Senay, L. Durak, and L. F. Chaparro, "A time-frequency division multiplexing communications system with hexagonal lattice structure," in Proceedings of 17th European Signal Processing Conference (EUSIPCO '09), Glasgow, Scotland, August 2009.

[8] A. Vahlin and N. Holte, "Optimal finite duration pulses for OFDM," IEEE Transactions on Communications, vol. 44, no. 1, pp. 10-14, 1996.

[9] D. Slepian, "Prolate spheroidal wave functions, fourier analysis and uncertainty. V: the discrete case," Bell System Technical Journal, vol. 57, no. 5, pp. 1371-1430, 1978.

[10] W. Kozek and A. F. Molisch, "Nonorthogonal pulseshapes for multicarrier communications in doubly dispersive channels," IEEE Journal on Selected Areas in Communications, vol. 16, no. 8, pp. 1579-1589, 1998.

[11] T. Strohmer and S. Beaver, "Optimal OFDM design for time-frequency dispersive channels," IEEE Transactions on Communications, vol. 51, no. 7, pp. 1111-1122, 2003.

[12] J. A. N. da Silva and M. L. R. de Campos, "Spectrally efficient UWB pulse shaping with application in orthogonal PSM," IEEE Transactions on Communications, vol. 55, no. 2, pp. 313322, 2007.

[13] J. Gomes and B. K. Mishra, "Orthogonal hermite pulses for indoor communication with UWB (S-V) channel," in Proceedings of the 1st InternationalConference on Computational Intelligence, Communication Systems and Networks, pp. 369373, Indore, India, July 2009.

[14] H. M. Ozaktas, Z. Zalevski, and M. A. Kutay, The Fractional Fourier Transform with Applications in Optics and Signal Processing, New York, NY, USA, John Wiley \& Sons, 2001. 